\title{
Valuation of Credit Default Swap with Counterparty Default Risk by Structural Model*
}

\author{
Jin Liang ${ }^{\mathbf{1}^{*}}$, Peng Zhou ${ }^{2}$, Yujing Zhou ${ }^{1}$, Junmei Ma ${ }^{3,4}$ \\ ${ }^{1}$ Department of Mathematics, Tongji University, Shanghai, China \\ ${ }^{2}$ Deloitte Touche Tomatsu CPA Ltd., Shanghai, China \\ ${ }^{3}$ Department of Applied Mathematics, Shanghai University of Finance and Economics, Shanghai, China \\ ${ }^{4}$ Department of Applied Mathematics, Tongji University, Shanghai, China \\ E-mail: liang_jin@tongji.edu.cn \\ Received June 19, 2010; revised November 18, 2010; accepted November 23, 2010
}

\begin{abstract}
This paper provides a methodology for valuing a credit default swap (CDS) with considering a counterparty default risk. Using a structural framework, we study the correlation of the reference entity and the counterparty through the joint distribution of them. The default event discussed in our model is associated to whether the minimum value of the companies in stochastic processes has reached their thresholds (default barriers). The joint probability of minimums of correlated Brownian motions solves the backward Kolmogorov equation, which is a two dimensional partial differential equation. A closed pricing formula is obtained. Numerical methodology, parameter analysis and calculation examples are implemented.
\end{abstract}

Keywords: CDS Spread, Counterparty Default Risk, Structural Model, PDE Method, Monte Carlo Calculation

\section{Introduction}

A vanilla credit default swap (CDS) is a kind of insurance against credit risk. The buyer of the CDS is the buyer of protection who pays a fixed fee or premium to the seller of protection for a period of time. If a certain pre-specified "credit event" occurs, the seller pays compensation to the buyer. The "credit event" can be a bankruptcy of a company, called the "reference entity", or a default of a bond or other debt issued by the reference entity. In this paper, the "credit event" also includes the default of the protection seller. If there is no credit event occurs during the term of the swap, the buyer continues to pay the premium until the CDS maturity.

A financial institution may use a CDS to transfer credit risk of a risky asset while continues to retain the legal ownership of the asset. As the rapid growth of the credit default swap market, credit default swaps on reference entity are more actively traded than bonds issued by the reference entities.

There are two primary types of models of default risk in the literature: structural models and reduced form (or

*This work is supported by National Basic Research Program of China (973 Program)2007CB814903. intensity) models. A structural model uses the evolution of a firm's structural variables, such as an asset and debt values, to determine the time of a default. Merton's model [1] is considered as the first structural model. In Merton's model, it is assumed that a company has a very simple capital structure where its debt has a face value of $D$ and maturity of time $T$, provides a zero coupon. Merton shows that the company's equity can be regarded as a European call option on its asset with a strike price of $D$ and maturity of $T$. A default occurs at $T$ if the option is not exercised. The second approach, within the structural framework, was introduced by Black-Cox [2] and Longstaff-Schwartz [3]. In this approach a default occurs as soon as the firm's asset value falls below a certain level. In contrast to the Merton approach, the default can occur at any time. Zhou [4,5] produces an analytic result for the default correlation between two firms by this model. Using this model, credit spread with jump is considered by Zhou [6].

Reduced form models do not care the relation between default and firm value in an explicit manner. In contrast to structural models, the time of default in intensity models is not determined via the value of the firm, but the 
first jump of an exogenously given jump process. The parameters governing the default hazard rate are inferred from market data. These models can incorporate correlations between defaults by allowing hazard rates to be stochastic and correlated with macroeconomic variables. Duffie-Singleton [7,8] and Lando [9] provide examples of research following this approach.

There have been many works on the pricing of credit default swaps. Hull - White [10] first considered the valuation of a vanilla credit default swap when there is no counterparty default risk. Their methodology is a twostage procedure. The first stage is to calculate the default probabilities at different future times from the yields on bonds issued by the reference entity. The second stage is to calculate the present value of both the expected future payoff and expected future payments on the CDS. They extended their study to the situation where there is possibility of counterparty default risk and obtained a pricing formula with Monte Carlo simulation [11]. They argued that if the default correlation between the protection seller and the reference entity is positive, the default of the counterparty will result in a positive replacement cost for the protection buyer.

Affection of the correlation on a CDS pricing remains interesting. The valuation of the credit default swap is based on computing the joint default probability of the reference entity and the counterparty (protection seller). Technically it is difficult because correlation between the entities involved in the contract is hard to deal with. Jarrow and Yildirim [12] obtained a closed form valuation formula for a CDS based on reduced form approach with correlated credit risk. In their model, the default intensity is assumed to be linear in the short interest rate. Jarrow and $\mathrm{Yu}$ [13] also assumed an inter-dependent default structure that avoided looping default and simplified the payoff structure where the seller's compensation was only made at the maturity of the swap. They discovered that a CDS may be significantly overpriced if the counterparty default probability was ignored. Yu [14] constructed the default processes from independent and identically distributed exponential random variables using the "total hazard" approach. He obtained an analytic expression of the joint distribution of default times when there were two or three firms in his model. Leung and Kwok [15] considered the valuation of a CDS with counterparty risk using a contagion model. In their model, if one firm defaults, the default intensity of another party will increase. They considered a more realistic scenario in which the compensation payment upon default of the reference entity was made at the end of the settlement period after default. They also extended their model to the three-firm situation.
More studies on different kinds of CDS, such as a basket reference entities, can be found from, e.x. [2, 15-23].

In this paper we develop a partial differential equation (PDE) procedure for valuing a credit default swap with counterparty default risk. In our model, a default event is supposed to occur at most one time, which means either reference entity or counterparty may default once. Our work is based on the structural framework, where the default event is associated to whether the minimum value of stochastic processes (value of the companies) have reached their thresholds (default barriers). Usually we choose the companies' liability as the thresholds [1,24]. We show that the joint probability of minimums of correlated Brownian motions solves the backward Kolmogorov equation, which is a two dimensional PDE with cross derivative term. This equation can be solved as a summation of Bessel and Sturm-Liouville eigenfunctions. The defaultable CDS studied in this paper, same as HullWhite's, is a special case. More complicated features of that kind of CDS are not considered.

The paper is organized as follows. In Section 2, we present a CDS spread expression. In Section 3, we establish a partial differential equation model which solves the joint probability distribution of two correlated companies used in Section 2 under the some assumptions. We obtain an explicit solution for this PDE. The main result of the closed form of the pricing the CDS then follows and shows in this section. Numerical calculation, example tests and parameter analysis for our model are collected in Section 4. We conclude the paper in Section 5.

\section{CDS Spread with Counterparty Default Risk}

In this section, first, we analyze how to value a CDS with counterparty default risk. Assume that party A holds a corporate bond with notional principal of $\$ 1$. To seek insurance against the default risk of the bond issuer (reference entity B), party A (CDS protection buyer) enters a CDS contract and makes a series of fixed, periodic payments of the CDS premium to party C (CDS protection seller) until the maturity, or until the credit event occurs. In exchange, party $\mathrm{C}$ promises to compensate party $\mathrm{A}$ for its loss if the credit event occurs. The amount of this compensation is usually the notional principal of the bond multiplied by $(1-R)$, where $R$ is the recovery rate, as a percentage of the notional. During the life time of the CDS, a risk-free interest is applied.

Assume that the default event, the risk-free interest rate and the recovery rate are mutually independent. Define, for the credit default swap, 
T: $\quad$ Maturity of the credit default swap;

$R: \quad$ Recovery rate on reference obligation;

$\bar{r}: \quad$ Risk neutral interest rate;

$w$ : Total payments per year made by the CDS buyer (party A) per $\$ 1$ of notional principal;

$\xi(t)$ : Risk neutral probability density of default by the reference entity and no default by the counterparty;

$\psi(t)$ : Risk neutral probability density of default by the counterparty and no default by the reference entity.

A vanilla CDS contract usually specifies two potential cash flow streams - a fixed premium leg and a contingent leg. On the premium leg side, the buyer of protection makes a series of fixed, periodic payments of the CDS premium until maturity or until a credit event occurs. On the contingent leg side, the protection seller makes a single payment in the case of the credit event. The value of the CDS contract to the protection buyer at any given point of time is the difference between the expected present value of the contingent leg, which is the protection buyer expects to receive, and that of the fixed leg, which he expects to pay, or

$$
\text { Value of } \begin{aligned}
C D S & =E[P V(\text { contingent leg })] \\
& -E[P V(\text { fixed premium leg })]
\end{aligned}
$$

Similar to the vanilla CDS, we assume that the payments are made at dates $t_{1}<t_{2}<\cdots<t_{n}=T$. Let $\Delta t$ be the time interval between payments dates, then the payment made every time is $w \Delta t$. In practice the payments are usually made quarterly, therefore $\Delta t=0.25$. The CDS payments cease when either the reference entity or the counterparty defaults. If a credit event occurs at time $\tau(0<\tau \leq T)$, denote the payments dates precisely before and after the default time $\tau$ by $t_{n_{\tau}}$ and $t_{n_{\tau}+1}$. When this credit event occurs exactly at one of the payments dates, let $t_{n_{\tau}}=\tau$. Then we have $t_{n_{\tau}} \leq \tau<t_{n_{\tau}+1}$.

First, we analyze the fixed premium leg side. As we assumed, the credit event occurs at most once. That is, there are three cases as follows.

Case 1 . the credit event is that the reference entity defaults at time $\tau$. Then the present value of all payments is

$$
w \sum_{i=1}^{n_{\tau}} \Delta t e^{-\bar{r}_{i}}+w\left(\tau-t_{n_{\tau}}\right) e^{-\bar{r} t_{n_{\tau}}+1}:=w \cdot a(\tau)+w \cdot e(\tau) .
$$

Case 2. the credit event is that the counterparty defaults at time $\tau$. Then there is no final accrued payment and the present value of all payments is

$$
w \sum_{i=1}^{n_{\tau}} \Delta t e^{-\bar{r}_{i}}=w \cdot a(\tau)
$$

Case 3. neither the reference entity nor the counterparty defaults prior to maturity time $T$. This time the present value of the payments is $w \cdot a(T)$.

Using the default probability densities of $\xi(t)$ and $\psi(t)$, the total expected present value of the premium leg is

$$
w \int_{0}^{T}[\xi(\tau) a(\tau)+\xi(\tau) e(\tau)+\psi(\tau) a(\tau)] d \tau+\pi w a(T) .
$$

On the contingent leg side, if the reference entity defaults at time $\tau$, the present value of the payoff form the CDS is given by

$$
(1-R) e^{-\bar{r}(\tau+\delta)},
$$

where $\delta$ is the liquidation period. The expected payoff is

$$
\int_{0}^{T} \xi(\tau)(1-R) e^{-\bar{r}(\tau+\delta)} d \tau .
$$

According to (1), the value of the CDS at time $t$ is

$$
\begin{aligned}
& \left(\int_{t}^{T} \xi(\tau)(1-R) e^{-\bar{r}(\tau+\delta)} d \tau\right) \\
& -\left(w \int_{t}^{T}[\xi(\tau) a(\tau)+\xi(\tau) e(\tau)+\psi(\tau) a(\tau)] d \tau+\pi w a(T)\right) .
\end{aligned}
$$

The value of the swap at origination must be equal to zero. The CDS spread $s$ is the value of $w$ which makes the value of the CDS equal to zero. Thus

$$
s=\frac{\int_{0}^{T} \xi(\tau)(1-R) e^{-\bar{r}(\tau+\delta)} d \tau}{\int_{0}^{T}[\xi(\tau) a(\tau)+\xi(\tau) e(\tau)+\psi(\tau) a(\tau)] d \tau+\pi a(T)} .
$$

The variable $s$ is referred as the credit default swap spread or the CDS spread. It is the total of the payments per year, as a percentage of the notional principal.

In expression (2), the joint probability densities are still unknown. We will focus on how to obtain these probability densities in following sections.

\section{Modelling and Solution}

In this section, we present several mathematical theorems which are necessary for the valuation of the CDS with the counterparty default risk. In order to describe the correlation between the reference entity and the counterparty, we study the joint probability distribution functions of the minimum values of two correlated Brownian motions.

The following are Basic Assumptions for our model.

1) Interest rate $r$ is constant;

2) Firm $i$ 's asset value $V_{i}(t)$ follows a geometric Brownian process with constant drift $\bar{r}$ and volatility $\sigma_{i}$ under the risk neutral measurement, 


$$
\frac{d V_{i}(t)}{V_{i}(t)}=\bar{r} d t+\sigma_{i} d W_{i}(t), i=1,2,
$$

where $\operatorname{cov}\left(d W_{1}(t), d W_{2}(t)\right)=\rho d t$ with $\rho$ being a constant;

3) Firm $i$ defaults as soon as its asset value $V_{i}(t)$ reaches the default barrier $D_{i}$. In this paper, we use the Black-Cox type default barrier, which is

$$
D_{i}(t)=F_{i} e^{-\gamma_{i} t},
$$

where $F_{i}$ and $\gamma_{i}$ are given constants respectively (see [2]);

4) The credit event occurs at most once.

\subsection{Default Probability}

Take $X_{i}(t)=\ln \left(\frac{V_{i}(t)}{V_{i}(0)} e^{-\gamma_{i} t}\right)$, then $X_{i}(0)=0$ and

$$
d X_{i}(t)=\mu_{i} d t+\sigma_{i} d W_{i}(t)
$$

where $\mu_{i}=r-\gamma_{i}-\frac{1}{2} \sigma_{i}^{2}$. The default barriers change to

$$
\begin{cases}\mathcal{L} u=\frac{\partial u}{\partial t}+\mu_{1} \frac{\partial u}{\partial x_{1}}+\mu_{2} \frac{\partial u}{\partial x_{2}}+\frac{1}{2} \sigma_{1}^{2} \frac{\partial^{2} u}{\partial x_{1}^{2}}+\frac{1}{2} \sigma_{2}^{2} \frac{\partial^{2} u}{\partial x_{2}^{2}}+\rho \sigma_{1} \sigma_{2} \frac{\partial^{2} u}{\partial x_{1} \partial x_{2}}=0, & \left(x_{1}, x_{2}, t\right) \in\left(m_{1},+\infty\right) \times\left(m_{2},+\infty\right) \times(0, T), \\ u\left(m_{1}, x_{2}, t\right)=1, & \left(x_{1}, x_{2}, t\right) \in\left(m_{1},+\infty\right) \times\left(m_{2},+\infty\right) \times(0, T), \\ u\left(x_{1}, m_{2}, t\right)=0, & \left(x_{1}, t\right) \in\left(m_{1},+\infty\right) \times(0, T), \\ u\left(x_{1}, x_{2}, T\right)=0, & \left(x_{1}, x_{2}\right) \in\left(m_{1},+\infty\right) \times\left(m_{2},+\infty\right),\end{cases}
$$

where $m_{1}, m_{2} \leq 0$.

Proof. Using Itô's formula (see, e.x. [25]), denote $X_{i}(t)=X_{i t}, i=1,2$,

$$
\begin{aligned}
u\left(X_{1 t}, X_{2 t}, t\right) & =u\left(x_{1}, x_{2}, 0\right) \\
& +\int_{0}^{t}\left(\frac{\partial u}{\partial s}+\mu_{1} \frac{\partial u}{\partial x_{1}}+\mu_{2} \frac{\partial u}{\partial x_{2}}+\frac{1}{2} \sigma_{1}^{2} \frac{\partial^{2} u}{\partial x_{1}^{2}}+\frac{1}{2} \sigma_{2}^{2} \frac{\partial^{2} u}{\partial x_{2}^{2}}+\rho \sigma_{1} \sigma_{2} \frac{\partial^{2} u}{\partial x_{1} \partial x_{2}}\right) d s \\
& +\int_{0}^{t} \sigma_{1} \frac{\partial u}{\partial x_{1}} d W_{1 s}+\int_{0}^{t} \sigma_{2} \frac{\partial u}{\partial x_{2}} d W_{2 s} .
\end{aligned}
$$

Assume that $u$ is the solution of backward Kolmogorov equation (4), so

$$
\begin{aligned}
\mathcal{L} u= & \frac{\partial u}{\partial s}+\mu_{1} \frac{\partial u}{\partial x_{1}}+\mu_{2} \frac{\partial u}{\partial x_{2}}+\frac{1}{2} \sigma_{1}^{2} \frac{\partial^{2} u}{\partial x_{1}^{2}} \\
& +\frac{1}{2} \sigma_{2}^{2} \frac{\partial^{2} u}{\partial x_{2}^{2}}+\rho \sigma_{1} \sigma_{2} \frac{\partial^{2} u}{\partial x_{1} \partial x_{2}} \\
= & 0 .
\end{aligned}
$$

Then

$$
u\left(X_{1 t}, X_{2 t}, t\right)=u\left(x_{1}, x_{2}, 0\right)+\int_{0}^{t} \sigma_{1} \frac{\partial u}{\partial x_{1}} d W_{1 s}
$$

$$
+\int_{0}^{t} \sigma_{2} \frac{\partial u}{\partial x_{2}} d W_{2 s}
$$

and

$$
E\left[u\left(X_{1 t}, X_{2 t}, t\right)\right]=u\left(x_{1}, x_{2}, 0\right) .
$$

Define the first passage time

$$
\tau=\inf \left\{s \mid \underline{X}_{1}(s) \leq m_{1}, \underline{X}_{2}(s)>m_{2}, s \geq 0\right\} .
$$

Let $t=\tau \wedge T$, we find (6) is

$$
u\left(x_{1}, x_{2}, 0\right)=E\left[u\left(X_{1 t}, X_{2 t}, t\right)\right]
$$




$$
\begin{aligned}
= & \int_{0}^{T} u\left(m_{1}, X_{2 s}, s\right) p\left(m_{1}, X_{2 s}, s ; x_{1}, x_{2}, 0\right) d s \\
& +\int_{0}^{T} u\left(X_{1 s}, m_{2}, s\right) p\left(X_{1 s}, m_{2}, s ; x_{1}, x_{2}, 0\right) d s \\
& +\int_{m_{1}}^{\infty} \int_{m_{2}}^{\infty} u\left(\xi_{1}, \xi_{2}, T\right) p\left(\xi_{1}, \xi_{2}, T ; x_{1}, x_{2}, 0\right) d \xi_{1} d \xi_{2},
\end{aligned}
$$

where $p\left(X_{1 t}, X_{2 t}, t ; x_{1}, x_{2}, 0\right)$ is the transition probability of being at state $\left(X_{1 t}, X_{2 t}\right)$ at time $t$, given that it starts at $\left(x_{1}, x_{2}\right)$ at time 0 .

Notice here, the above equation is also held for $u\left(x_{1}, x_{2}, t_{0}\right), 0<t_{0}<t$, if only change the low limit of the integration.

Because of the boundary and final-time conditions in (4), we get

$$
u\left(x_{1}, x_{2}, 0\right)=E\left[u\left(X_{1 t}, X_{2 t}, t\right)\right]=\int_{0}^{T} p\left(m_{1}, X_{2 s}, s ; x_{1}, x_{2}, 0\right) d s .
$$

According to the definition of $p\left(m_{1}, X_{2 s}, s ; x_{1}, x_{2}, 0\right)$ defined at the end of (7), $u\left(x_{1}, x_{2}, 0\right)$ is the probability defined in (3) at time 0 .

Now, let us solve PDE (4).

First, we make the following transformation to eliminate the drift terms. Let $\tau=T-t$ and

$$
u\left(x_{1}, x_{2}, \tau\right)=e^{a_{1} x_{1}+a_{2} x_{2}+b \tau} p\left(x_{1}, x_{2}, \tau\right),
$$

where

$$
\begin{aligned}
& a_{1}=\frac{\rho \mu_{2} \sigma_{1}-\mu_{1} \sigma_{2}}{\left(1-\rho^{2}\right) \sigma_{1}^{2} \sigma_{2}}, \quad a_{2}=\frac{\rho \mu_{1} \sigma_{2}-\mu_{2} \sigma_{1}}{\left(1-\rho^{2}\right) \sigma_{1} \sigma_{2}^{2}}, \\
& b=\mu_{1} a_{1}+\mu_{2} a_{2}+\frac{1}{2} \sigma_{1}^{2} \mu_{1}^{2}+\rho \sigma_{1} \sigma_{2} \mu_{1} \mu_{2}+\frac{1}{2} \sigma_{2}^{2} \mu_{2}^{2} .
\end{aligned}
$$

Then $p\left(x_{1}, x_{2}, \tau\right)$ satisfies

$$
\left\{\begin{array}{l}
\frac{\partial p}{\partial \tau}-\frac{1}{2} \sigma_{1}^{2} \frac{\partial^{2} p}{\partial x_{1}^{2}}-\frac{1}{2} \sigma_{2}^{2} \frac{\partial^{2} p}{\partial x_{2}^{2}}-\rho \sigma_{1} \sigma_{2} \frac{\partial^{2} p}{\partial x_{1} \partial x_{2}}=0 \\
p\left(m_{1}, x_{2}, \tau\right)=e^{-\left(a_{1} m_{1}+a_{2} x_{2}+b \tau\right)} \\
p\left(x_{1}, m_{2}, \tau\right)=0 \\
p\left(x_{1}, x_{2}, 0\right)=0
\end{array}\right.
$$

Next, we eliminate the cross-partial derivative and normalize the Brownian motions by a suitable transformation of coordinates, this idea was introduced by $\mathrm{He}$ etc. ([26]. Define new coordinates $z_{1}$ and $z_{2}$ as the following

$$
\begin{aligned}
& z_{1}=-\frac{1}{\sqrt{1-\rho^{2}}}\left[\left(\frac{x_{1}-m_{1}}{\sigma_{1}}\right)-\rho\left(\frac{x_{2}-m_{2}}{\sigma_{2}}\right)\right], \\
& z_{2}=\frac{x_{2}-m_{2}}{\sigma_{2}} .
\end{aligned}
$$

Then $q\left(z_{1}\left(x_{1}, x_{2}\right), z_{2}\left(x_{1}, x_{2}\right), \tau\right)=p\left(x_{1}, x_{2}, \tau\right)$ satisfies

$$
\left\{\begin{array}{l}
\frac{\partial q}{\partial \tau}=\frac{1}{2}\left(\frac{\partial^{2} q}{\partial z_{1}^{2}}+\frac{\partial^{2} q}{\partial z_{2}^{2}}\right) \\
q\left(L_{1}, \tau\right)=0 \\
q\left(L_{2}, \tau\right)=e^{-\left(a_{1} m_{1}+a_{2}\left(\sigma_{2} z_{2}+m_{2}\right)+b \tau\right)} \\
q\left(z_{1}, z_{2}, 0\right)=0
\end{array}\right.
$$

where

$$
L_{1}=\left\{\left(z_{1}, z_{2}\right) \mid z_{2}=0\right\}, L_{2}=\left\{\left(z_{1}, z_{2}\right) \mid z_{2}=\frac{\sqrt{1-\rho^{2}}}{\rho} z_{1}\right\} \text {. }
$$

Because the boundary conditions are more conveniently expressed in polar coordinates, we introduce $(r, \theta)$ corresponding to $\left(z_{1}, z_{2}\right)$ as

$$
r=\sqrt{z_{1}^{2}+z_{2}^{2}}, \quad \tan \theta=\frac{z_{2}}{z_{1}},
$$

thus $\beta \in(0, \pi / 2)$ and obtain $q(r, \theta, \tau)$ satisfies

$$
\left\{\begin{array}{l}
\frac{\partial q}{\partial \tau}=\frac{1}{2}\left(\frac{\partial^{2} q}{\partial r^{2}}+\frac{1}{r} \frac{\partial q}{\partial r}+\frac{1}{r^{2}} \frac{\partial^{2} q}{\partial \theta^{2}}\right), \\
q(r, 0, \tau)=0, \\
q(r, \beta, \tau)=e^{-\left(a_{1} m_{1}+a_{2}\left(\sigma_{2} r \sin \beta+m_{2}\right)+b \tau\right)}, \\
q(r, \theta, 0)=0 .
\end{array}\right.
$$

Define a new function

$$
f(r, \theta, \tau)=\frac{\theta}{\beta} e^{-\left(a_{1} m_{1}+a_{2}\left(\sigma_{2} r \sin \beta+m_{2}\right)+b \tau\right)},
$$

then $g(r, \theta, \tau)=q(r, \theta, \tau)-f(r, \theta, \tau)$ solves

$$
\left\{\begin{array}{l}
\frac{\partial g}{\partial \tau}-\frac{1}{2}\left(\frac{\partial^{2} g}{\partial r^{2}}+\frac{1}{r} \frac{\partial g}{\partial r}+\frac{1}{r^{2}} \frac{\partial^{2} g}{\partial \theta^{2}}\right)=b f(r, \theta, \tau), \\
g(r, 0, \tau)=0, \\
g(r, \beta, \tau)=0, \\
g(r, \theta, 0)=-f(r, \theta, 0) .
\end{array}\right.
$$

In order to solve PDE (15), we consider the Green's function $G\left(r, \theta, \tau ; r_{0}, \theta_{0}, \tau_{0}\right)$ of this problem, which satisfies

$$
\left\{\begin{array}{l}
\frac{\partial G}{\partial \tau}=\frac{1}{2}\left(\frac{\partial^{2} G}{\partial r^{2}}+\frac{1}{r} \frac{\partial G}{\partial r}+\frac{1}{r^{2}} \frac{\partial^{2} G}{\partial \theta^{2}}\right), \\
G(r, 0, \tau)=G(r, \beta, \tau)=0, \\
G\left(r, \theta, \tau_{0}\right)=\delta\left(r-r_{0}\right) \delta\left(\theta-\theta_{0}\right) .
\end{array}\right.
$$

Lemma 2 The solution of PDE (16) is

$$
G\left(r, \theta, \tau ; r_{0}, \theta_{0}, \tau_{0}\right)=\frac{2 r_{0}}{\beta\left(\tau-\tau_{0}\right)} .
$$




$$
\sum_{n=1}^{\infty} e^{-\frac{r^{2}+r_{0}^{2}}{2\left(\tau-\tau_{0}\right)}} \sin \left(\frac{n \pi}{\beta} \theta_{0}\right) \sin \left(\frac{n \pi}{\beta} \theta\right) I_{\frac{n \pi}{\beta}}\left(\frac{r r_{0}}{\tau-\tau_{0}}\right) .
$$

Proof. We try to find separable solutions to this equation in the form of

$$
G(r, \theta, \tau)=M(r, \theta) T(\tau)
$$

Plugging (18) into (16), we find that

$$
M(r, \theta) T^{\prime}(\tau)=\frac{T(\tau)}{2}\left(\frac{\partial^{2} M}{\partial r^{2}}+\frac{1}{r} \frac{\partial M}{\partial r}+\frac{1}{r^{2}} \frac{\partial^{2} M}{\partial \theta^{2}}\right) .
$$
find

Divide the previous equation by $M(r, \theta) T(\tau)$, we

$$
\frac{T^{\prime}(\tau)}{T(\tau)}=\frac{1}{2 M(r, \theta)}\left(\frac{\partial^{2} M}{\partial r^{2}}+\frac{1}{r} \frac{\partial M}{\partial r}+\frac{1}{r^{2}} \frac{\partial^{2} M}{\partial \theta^{2}}\right)=-\frac{\lambda^{2}}{2} .
$$

Since the left side of (19) is a function of $\tau$ and the right side is a function of $r$ and $\theta$, so it must be a constant. Denote this constant by $-\frac{\lambda^{2}}{2}$ and we have

$$
T(\tau)=K e^{-\frac{1}{2} \lambda^{2} \tau} .
$$

On the other hand, $M(r, \theta)$ satisfies equation

$$
\left\{\begin{array}{l}
\frac{\partial^{2} M}{\partial r^{2}}+\frac{1}{r} \frac{\partial M}{\partial r}+\frac{1}{r^{2}} \frac{\partial^{2} M}{\partial \theta^{2}}+\lambda^{2} M=0 \\
M(r, 0)=M(r, \beta)=0 .
\end{array}\right.
$$

This is a Sturm-Liouville problem. We try to find separable solutions in the form of $M(r, \theta)=R(r) \Theta(\theta)$. Plugging this into (16) we get

$$
r^{2} \frac{R^{\prime \prime}}{R}+r \frac{R^{\prime}}{R}+\lambda^{2} r^{2}=-\frac{\Theta^{\prime \prime}}{\Theta},
$$

with boundary conditions

$$
R(r) \Theta(0)=R(r) \Theta(\beta)=0 .
$$

Let $\Theta^{\prime \prime} / \Theta=-k^{2}$, then $\Theta(\theta)$ solves

$$
\left\{\begin{array}{l}
\Theta^{\prime \prime}+k^{2} \Theta=0, \\
\Theta(0)=\Theta(\beta)=0 .
\end{array}\right.
$$

It is easy to see that

$$
\Theta(\theta)=A \sin k \theta+B \cos k \theta .
$$

Considering the boundary conditions, we have $B=0$ and $A \sin k \beta=0$. Because $\Theta(\theta)$ is non-zero solution, we know that $A \neq 0$ and

$$
k_{n}=\frac{n \pi}{\beta}, \quad n=1,2, \cdots .
$$

Thus the eigenfunctions consistent with the boundary conditions are

$$
\Theta_{\lambda}(\theta)=C_{n}(\lambda) \sin \frac{n \pi \theta}{\beta}, \quad n=1,2, \cdots .
$$

Finally consider the radial part of the solution $R(r)$ which satisfies

$$
r^{2} R^{\prime \prime}+r R^{\prime}+\left(\lambda^{2} r^{2}-k_{n}^{2}\right) R=0 .
$$

Denoting $\zeta=\lambda r$, we get the standard form of Bessel's equation

$$
\zeta^{2} \frac{\mathrm{d}^{2} R}{\mathrm{~d} \zeta^{2}}+\zeta \frac{\mathrm{d} R}{\mathrm{~d} \zeta}+\left(\zeta^{2}-k_{n}^{2}\right) R=0 .
$$

The well known fundamental solutions of this Bessel's equation is

$$
J_{k_{n}}(x)=\sum_{i=0}^{\infty} \frac{(-1)^{i}}{\Gamma\left(k_{n}+i+1\right) \Gamma(i+1)}\left(\frac{x}{2}\right)^{2 i+k_{n}}
$$

and

$$
Y_{k_{n}}(x)=\lim _{p \rightarrow k_{n}} \frac{J_{p}(x) \cos (p \pi)-J_{-p}(x)}{\sin (p \pi)} .
$$

Since $Y_{k_{n}}(0)$ diverges and we require $R(0)$ to be bounded, the solution $Y_{k_{n}}(y)$ is not permitted. Hence the general radial part of the solution is

$$
R_{\lambda, n}(r)=J_{k_{n}}(\lambda r) \text {. }
$$

Sum up $R_{\lambda, n}(r) \Theta_{\lambda, n}(\theta)$ over $n$, we have

$$
\begin{aligned}
M_{\lambda}(r, \theta) & =\sum_{n=1}^{\infty} R_{\lambda, n}(r) \Theta_{\lambda, n}(\theta) \\
& =\sum_{n=1}^{\infty} C_{n}(\lambda) J_{\frac{n \pi}{\beta}}(\lambda r) \sin \left(\frac{n \pi}{\beta} \theta\right) .
\end{aligned}
$$

Then

$$
\begin{aligned}
G_{\lambda}(r, \theta, \tau) & =M_{\lambda}(r, \theta) T_{\lambda}(\tau) \\
& =\sum_{n=1}^{\infty} K C_{n}(\lambda) e^{-\frac{\lambda^{2}}{2} \tau} J_{\frac{n \pi}{\beta}}(\lambda r) \sin \left(\frac{n \pi}{\beta} \theta\right) .
\end{aligned}
$$

Because $K$ is a constant, we can define $A_{n}(\lambda)=$ $K C_{n}(\lambda)$. Integral the previous equation over $\lambda$, we obtain the general solution to $\operatorname{PDE}(16)$ for $G(r, \theta, \tau)$ as

$$
G(r, \theta, \tau)=\int_{0}^{\infty} \sum_{n=1}^{\infty} A_{n}(\lambda) e^{-\frac{\lambda^{2}}{2} \tau} J_{\frac{n \pi}{\beta}}(\lambda r) \sin \left(\frac{n \pi}{\beta} \theta\right) d \lambda .
$$

Now we try to find the coefficient $A_{n}(\lambda)$ which fit the initial condition $G\left(r, \theta, \tau_{0}\right)=\delta\left(r-r_{0}\right) \delta\left(\theta-\theta_{0}\right)$. Multiply the previous equation at $\tau=\tau_{0}$ by $\sin \left(\frac{m \pi}{\beta} \theta\right)$ and integrate over $\theta$, we find 


$$
\delta\left(r-r_{0}\right) \sin \left(\frac{m \pi}{\beta} \theta_{0}\right)=\frac{\beta}{2} \int_{0}^{\infty} A_{m}(\lambda) e^{-\frac{\lambda^{2}}{2} \tau_{0}} J_{\frac{m \pi}{\beta}}(\lambda r) \mathrm{d} \lambda .
$$

Noticing the completeness relation of

$$
\int_{0}^{\infty} x J_{s}(a x) J_{s}(b x) \mathrm{d} x=\frac{1}{a} \delta(a-b),
$$

multiply equation (24) by $r J_{\frac{m \tau}{\beta}}\left(\lambda^{\prime} r\right)$ and integrate over $r$

$$
A_{m}\left(\lambda^{\prime}\right)=\frac{2 \lambda^{\prime} r_{0}}{\beta} e^{\frac{\lambda^{\prime 2}}{2} \tau_{0}} \sin \left(\frac{m \pi}{\beta} \theta_{0}\right) J_{\frac{m \pi}{\beta}}\left(\lambda^{\prime} r_{0}\right) .
$$

Plugging this expression into $G(r, \theta, \tau)$ (23), we get

$$
\begin{aligned}
& G(r, \theta, \tau)=\int_{0}^{\infty}\left(\frac{2 \lambda r_{0}}{\beta}\right) . \\
& \sum_{n=1}^{\infty} e^{-\frac{\lambda^{2}}{2}\left(\tau-\tau_{0}\right)} \sin \left(\frac{m \pi}{\beta} \theta_{0}\right) \sin \left(\frac{n \pi}{\beta} \theta\right) J_{\frac{n \pi}{\beta}}\left(\lambda r_{0}\right) J_{\frac{n \pi}{\beta}}(\lambda r) d \lambda .
\end{aligned}
$$

Using the fact [27] that

$$
\int_{0}^{\infty} x e^{-c^{2} x^{2}} J_{s}(a x) J_{s}(b x) d x=\frac{1}{2 c^{2}} e^{-\frac{a^{2}+b^{2}}{4 c^{2}}} I_{s}\left(\frac{a b}{2 c^{2}}\right),
$$

(25) can be simplified into (17).

With Green's function $G\left(r, \theta, \tau ; r_{0}, \theta_{0}, \tau_{0}\right)$ and the boundary and initial conditions, the solution of PDE (15) can be expressed as

$$
\begin{aligned}
q(r, \theta, \tau)= & \int_{0}^{\tau} d \tau_{0} \iint_{F} G\left(r, \theta, \tau ; r_{0}, \theta_{0}, \tau_{0}\right) b f\left(r_{0}, \theta_{0}, \tau_{0}\right) d r_{0} d \theta_{0} \\
& -\iint_{F} G\left(r, \theta, \tau ; r_{0}, \theta_{0}, 0\right) f\left(r_{0}, \theta_{0}, 0\right) d r_{0} d \theta_{0},
\end{aligned}
$$

where

$$
\begin{aligned}
& F=\{(r, \theta) \mid 0 \leq r<+\infty, 0 \leq \theta \leq \beta\}, \\
& f(r, \theta, \tau)=\frac{\theta}{\beta} e^{-\left(a_{1} m_{1}+a_{2}\left(\sigma_{2} r \sin \beta+m_{2}\right)+b \tau\right)} .
\end{aligned}
$$

Then solution of PDE (13) is

$$
q(r, \theta, \tau)=g(r, \theta, \tau)+f(r, \theta, \tau) .
$$

Returning to the original coordinates and variables $\left(x_{1}, x_{2}, t\right)$, we get

$$
\begin{aligned}
u\left(x_{1}, x_{2}, t\right) & =e^{a_{1} x_{1}+a_{2} x_{2}+b(T-t)} p\left(x_{1}, x_{2}, T-t\right) \\
& =e^{a_{1} x_{1}+a_{2} x_{2}+b(T-t)} q\left(z_{1}, z_{2}, T-t\right) \\
& =e^{a_{1} x_{1}+a_{2} x_{2}+b(T-t)} q(r, \theta, T-t),
\end{aligned}
$$

where $z_{1}, z_{2}, r, \theta$ are defined in (9), (10) and (12).
That is, we have

Theorem 1 The solution of the initial boundary problem (4) has a closed form solution (29) associated by (26), (14) and (17).

By now, we have already obtained the probability of that company 1 defaults and company 2 does not defaults between time $t$ and $T$. Change $t$ into $0, T$ into $t$, here comes the probability between time 0 and $t$.

In order to obtain the probability $v\left(x_{1}, x_{2}, t\right)$ of that company 2 defaults and company 1 does not default, we only need to change the positions of the parameters of the two companies such as $\sigma_{i}, F_{i}, \gamma_{i}, V_{i}(0)$ in $u\left(x_{1}, x_{2}, t\right)$.

Now apply our result to the spread Formula (2) when $\delta=0$. In the valuation formula of (2), what we need are the default probability density functions of $\xi(\tau)$ and $\psi(\tau)$ while we only have the probability functions. Therefore, we need to modify (2). In fact, notice that $a(\tau)$ and $e(\tau)$ are piecewise continuous functions and on every piece,

$$
a(\tau)^{\prime}=0, \quad e(\tau)^{\prime}=\left(\left(\tau-t_{n_{\tau}}\right) e^{-r \tau}\right)^{\prime}=e^{-r \tau}-r e(\tau) .
$$

Integrate the numerator and denominator of (2) by parts, and we have

$$
\begin{aligned}
\text { numerator }= & \int_{0}^{T}(1-R) e^{-r \tau} d \Xi(\tau) \\
= & (1-R)\left(e^{-r T} \Xi(T)+r \int_{0}^{T} e^{-r \tau} \Xi(\tau) d \tau\right), \\
\text { denominator } & =\int_{0}^{T}(a(\tau)+e(\tau)) d \Xi(\tau) \\
& +\int_{0}^{T} a(\tau) d \Psi(\tau)+a(T) \pi \\
& =\sum_{i=1}^{n} \int_{t_{i-1}}^{t_{i}}(a(\tau)+e(\tau)) d \Xi(\tau) \\
& +\sum_{i=1}^{n} \int_{t_{i-1}}^{t_{i}} a(\tau) d \Psi(\tau)+a(T) \pi \\
& =\sum_{i=1}^{n}\left[\left.(a(\tau)+e(\tau)) \Xi(\tau)\right|_{t_{i-1}} ^{t_{i}}\right. \\
& \left.-\int_{t_{i-1}}^{t_{i}} \Xi(\tau)\left(e^{-r \tau}-r e(\tau)\right) d \tau\right] \\
& +\left.\sum_{i=1}^{n} a(\tau) \Psi(\tau)\right|_{t_{i-1}} ^{t_{i}}+a(T) \pi,
\end{aligned}
$$

where

$$
\Xi(\tau)=u(\cdot,, \tau) \text { and } \Psi(\tau)=v(\cdot,, \tau),
$$

for $u$ and $v$ are solved in this subsection.

\subsection{Survival Probability $\pi\left(x_{1}, x_{2}, t\right)$}

To calculate the credit default swap spread $s$, we still need to study the joint survival probability of $\pi\left(x_{1}, x_{2}, t\right)$ for $x_{1}>m_{1}, x_{2}>m_{2}$, 
$\pi\left(x_{1}, x_{2}, t\right)$

$=\operatorname{Prob}\left(\underline{X}_{1}(T)>m_{1}, \underline{X}_{2}(T)>m_{2} \mid X_{1}(t)=x_{1}, X_{2}(t)=x_{2}\right)$.

$$
\begin{cases}\mathcal{L} \pi=\frac{\partial \pi}{\partial t}+\mu_{1} \frac{\partial \pi}{\partial x_{1}}+\mu_{2} \frac{\partial \pi}{\partial x_{2}} & +\frac{1}{2} \sigma_{1}^{2} \frac{\partial^{2} \pi}{\partial x_{1}^{2}}+\frac{1}{2} \sigma_{2}^{2} \frac{\partial^{2} \pi}{\partial x_{2}^{2}}+\rho \sigma_{1} \sigma_{2} \frac{\partial^{2} \pi}{\partial x_{1} \partial x_{2}}=0, \\ \pi\left(m_{1}, x_{2}, t\right)=0, & \left(x_{1}, x_{2}, t\right) \in\left(m_{1},+\infty\right) \times\left(m_{2},+\infty\right) \times(0, T), \\ \pi\left(x_{1}, m_{2}, t\right)=0, & \left(x_{2}, t\right) \in\left(m_{2},+\infty\right) \times(0, T), \\ \pi\left(x_{1}, x_{2}, T\right)=1, & \left(x_{1}, x_{2}\right) \in\left(m_{1},+\infty\right) \times(0, T),\end{cases}
$$

where $m_{1}, m_{2} \leq 0$.

In the previous section, we get the solutions to these two PDEs in the domain $\left(m_{1},+\infty\right) \times\left(m_{2},+\infty\right) \times(0, T)$,

$$
\left\{\begin{array}{l}
\mathcal{L} u=\frac{\partial u}{\partial t}+\mu_{1} \frac{\partial u}{\partial x_{1}}+\mu_{2} \frac{\partial u}{\partial x_{2}}+\frac{1}{2} \sigma_{1}^{2} \frac{\partial^{2} u}{\partial x_{1}^{2}}+\frac{1}{2} \sigma_{2}^{2} \frac{\partial^{2} u}{\partial x_{2}^{2}}+\rho \sigma_{1} \sigma_{2} \frac{\partial^{2} u}{\partial x_{1} \partial x_{2}}=0, \\
u\left(m_{1}, x_{2}, t\right)=1, \\
u\left(x_{1}, m_{2}, t\right)=0, \\
u\left(x_{1}, x_{2}, T\right)=0,
\end{array}\right.
$$

and

$$
\left\{\begin{array}{l}
\mathcal{L} v=\frac{\partial v}{\partial t}+\mu_{1} \frac{\partial v}{\partial x_{1}}+\mu_{2} \frac{\partial v}{\partial x_{2}}+\frac{1}{2} \sigma_{1}^{2} \frac{\partial^{2} v}{\partial x_{1}^{2}}+\frac{1}{2} \sigma_{2}^{2} \frac{\partial^{2} v}{\partial x_{2}^{2}}+\rho \sigma_{1} \sigma_{2} \frac{\partial^{2} v}{\partial x_{1} \partial x_{2}}=0 \\
v\left(m_{1}, x_{2}, t\right)=0 \\
v\left(x_{1}, m_{2}, t\right)=1, \\
v\left(x_{1}, x_{2}, T\right)=0
\end{array}\right.
$$

where $m_{1}, m_{2} \leq 0$.

Compare the boundary and final conditions of PDE (31), (32), (33), the solution to (31) can be written as the linear combination of the other two

$$
\pi\left(x_{1}, x_{2}, t\right)=1-u\left(x_{1}, x_{2}, t\right)-v\left(x_{1}, x_{2}, t\right) .
$$

$$
s=\frac{(1-R)\left(e^{-r T} \Xi(T)+r \int_{0}^{T} e^{-r \tau} \Xi(\tau) d \tau\right)}{\sum_{i=1}^{n}\left[\left.(a(\tau)+e(\tau)) \Xi(\tau)\right|_{t_{i-1}} ^{t_{i}}-\int_{t_{i-1}}^{t_{i}} \Xi(\tau)\left(e^{-r \tau}-r e(\tau)\right) d \tau+\left.a(\tau) \Psi(\tau)\right|_{t_{i-1}} ^{t_{i}}\right]+a(T) \pi} .
$$

Remark 1 It is a special case of our model that the $C D S$ with the counterparty default when the correlation of the counterparty and reference entity are independent.

Remark 2 The same method can be used to the pricing the CDS for a basket reference entities. In this case, the PDE model is simpler as the boundaries condition are all equal to 0 . So that, it has no problem caused by the singularity near $(0,0)$. However, if the basket has a big number of reference entities, the closed form solution of the PDE is difficult to be obtained.

\subsection{Main Result}

Combine the previous two subsection, we obtain the all probabilities required in Formula (36). Therefore we ob-
Set $t=0$, we get the probability of $\pi$ which was defined in Section 2

$$
\pi=\pi\left(x_{1}, x_{2}, 0\right)=1-\Xi(0)-\Psi(0) .
$$

tain the main theorem of this paper presented as follows:

Theorem 2 (main theorem) Under the Basic Assumption (1)-(4), the credit default swap spread with counterparty default risk is given by (36), where, in the formula, the probability $\Xi(\cdot)$ are given by (30) solving the problem (4); $\Psi(\cdot)$ are solved as $\Xi(\cdot)$ in the same way; $\pi$ is given by (35).

\section{Numerical Analysis}

So far, we have derived the three probabilities in Section 2. With these, we can calculate the CDS spread by (29).

Even though we have a so called closed or semi-closed form solution, but the calculation of the form is still not trivial. The expression of the form includes integration 
and infinite serial, as well as a special function. The direct calculation is not easy to undertaken and the result is usually not satisfying. This because that the value of the integrand concentrates in a very small area and this area is moving as the change of the time $t$. So that, the difference approximation, in general, will make the result value very small.

Here we introduce an algorithm of Monte Carlo method to evaluate the form (29). It sounds that there is no difference from the one to calculate CDS spread by direct Monte Carlo method, however, it is really different with and without the closed form solution. We will see it in the later.

Using Monte Carlo to calculate the closed form, in fact, we only need to know how to calculate the first integration of the Formula (26). The steps to do it are as follows:

1) Representing the integration with the exception of the integrand.

Take $\widetilde{f}\left(r_{0}, \theta_{0}, \tau_{0}\right) \triangleq c f\left(r_{0}, \theta_{0}, \tau_{0}\right) e^{-\bar{A} \sigma_{2} r_{0} \sin \beta} \quad$ as a density function, where $\bar{A}=\frac{\mu_{1} \sigma_{2}+\mu_{2} \sigma_{1}}{\sqrt{1-\rho^{2}} \sigma_{1} \sigma_{2}}, c$ is a constant such that

$$
c \int_{0}^{\tau} d \tau_{0} \iint_{F} f\left(r_{0}, \theta_{0}, \tau_{0}\right) e^{-\bar{A} \sigma_{2} r_{0} \sin \beta} d r_{0} d \theta_{0}=1,
$$

where $f\left(r_{0}, \theta_{0}, \tau_{0}\right)$, which is non-negative as $\beta \in(0, \pi / 2)$, is defined in (28). By simple calculation, we obtain

$$
c=\frac{2 b\left(a_{2}+\bar{A}\right) \sigma_{2} \sin \beta}{\beta e^{-\left(a_{1} m_{1}+a_{2} m_{2}\right)}\left(1-e^{-b \tau}\right)} \text {. }
$$

Now rewrite the integration, $E$ is measured respect to $\widetilde{f}$ :

$$
\begin{aligned}
& \int_{0}^{\tau} d \tau_{0} \iint_{F} G\left(r, \theta, \tau ; r_{0}, \theta_{0}, \tau_{0}\right) b f\left(r_{0}, \theta_{0}, \tau_{0}\right) d r_{0} d \theta_{0} \\
& =E\left[\frac{b}{c} G\left(r, \theta, \tau ; r_{0}, \theta_{0}, \tau_{0}\right) e^{\bar{A} \sigma_{2} r_{0} \sin \beta}\right],
\end{aligned}
$$

where $G$ is defined in (25).

2) Random numbers fetching.

In our case, the three-dimensional random $(X, Y, Z)$ has a joint density $\widetilde{f}\left(r_{0}, \theta_{0}, \tau_{0}\right)$. We sample this random variable $\left(X_{i}, Y_{i}, Z_{i}\right)$ from $U^{3}(0,1)$, for $i=1,2, \cdots$, in the following way:

a) First, the marginal density function respect to $r_{0}$ is

$$
\begin{aligned}
\tilde{f}_{1}\left(r_{0}\right) & =\int_{0}^{\tau} \int_{0}^{\beta} \tilde{f}\left(r_{0}, \theta_{0}, \tau_{0}\right) d \tau_{0} d \theta_{0} \\
& =\left(a_{2}+\bar{A}\right) \sigma_{2} \sin \beta e^{-\left(a_{2}+\bar{A}\right) \sigma_{2} r_{0} \sin \beta},
\end{aligned}
$$

and the marginal distribution function is

$$
\begin{aligned}
F_{1}\left(r_{0}\right) & =\int_{0}^{r_{0}}\left(a_{2}+\bar{A}\right) \sigma_{2} \sin \beta e^{-\left(a_{2}+\bar{A}\right) \sigma_{2} u \sin \beta} d u \\
& =1-e^{-\left(a_{2}+\bar{A}\right) \sigma_{2} r_{0} \sin \beta} .
\end{aligned}
$$

Then generate uniform random number $U_{1 i}$ and set $X_{i}=-\ln \left(1-U_{1 i}\right) /\left(a_{2}+\bar{A}\right) \sigma_{2} \sin \beta$.

b) Secondly, for given $X=r_{0}$, the conditional density function

$$
\tilde{f}\left(\theta_{0}, \tau_{0} \mid r_{0}\right)=\frac{\tilde{f}\left(r_{0}, \theta_{0}, \tau_{0}\right)}{\tilde{f}_{1}\left(r_{0}\right)}=\frac{2 b \theta_{0} e^{-b \tau_{0}}}{\beta^{2}\left(1-e^{-b \tau}\right)} .
$$

The marginal density function respect to $\theta_{0}$ is

$$
\tilde{f}_{2}\left(\theta_{0} \mid r_{0}\right)=\int_{0}^{\beta} \tilde{f}\left(\theta_{0}, \tau_{0} \mid r_{0}\right) d \tau_{0}=\frac{2 \theta_{0}}{\beta^{2}},
$$

and its distribution function is

$$
\tilde{F}_{2}\left(\theta_{0} \mid r_{0}\right)=\int_{0}^{\theta_{0}} \frac{2 u}{\beta^{2}} d u=\frac{\theta_{0}^{2}}{\beta^{2}} .
$$

Then generate uniform random number $U_{2 i}$ and set $Y_{i}=\sqrt{\beta^{2} U_{2 i}}$.

c) Thirdly, the joint marginal distribution function with respect to $r_{0}$ and $\theta_{0}$ is

$$
\begin{aligned}
\tilde{f}_{12}\left(r_{0}, \theta_{0}\right) & =\int_{0}^{\tau} \tilde{f}\left(r_{0}, \theta_{0}, \tau_{0}\right) d \tau_{0} \\
& =\frac{2 \theta_{0}}{\beta^{2}}\left(a_{2}+\bar{A}\right) \sigma^{2} \sin \beta e^{-\left(a_{2}+\bar{A}\right) \sigma^{2} r_{0} \sin \beta},
\end{aligned}
$$

then for given $X=r_{0}, Y=\theta_{0}$, the conditional density function $\tilde{f}_{3}\left(\tau_{0} \mid r_{0}, \theta_{0}\right)=\frac{\tilde{f}\left(r_{0}, \theta_{0}, \tau_{0}\right)}{f_{12}\left(r_{0}, \theta_{0}\right)}=\frac{b e^{-b \tau_{0}}}{1-e^{-b \tau}}$, and its distribution function is $\tilde{F}_{3}\left(\tau_{0} \mid r_{0}, \theta_{0}\right)=\frac{1-e^{-b \tau_{0}}}{1-e^{-b \tau}}$. Then generate uniform random number $U_{3 i}$ and set $Z_{i}=-\frac{1}{b} \ln \left(1-\left(1-e^{-b \tau}\right) U_{3 i}\right)$.

Therefore we generate the $i$ th random sample $\left(X_{i}, Y_{i}, Z_{i}\right)$ with density function $\tilde{f}\left(r_{0}, \theta_{0}, \tau_{0}\right)$.

$3)$ By the method above, obtain three-dimensional random sample $\left(X_{i}, Y_{i}, Z_{i}\right)$, then replace $\left(r_{0}, \theta_{0}, \tau_{0}\right)$ and put it into the integrand

$$
\frac{b}{c} G\left(r, \theta, \tau ; r_{0}, \theta_{0}, \tau_{0}\right) e^{\bar{A} \sigma_{2} r_{0} \sin \beta} .
$$

For $i=1,2, \cdots, n$, repeat the process $n$ times (e.g. $n=1000,10000 \cdots$ as required), then find the mean value, to find approximated the expectation.

It may argue that if use Monte Carlo method, why just simulate directly on the original Formula (2)? The Figure 1 can answer this question.

Consider practical examples. Assuming there are two companies $\mathrm{B}$ and $\mathrm{C}$ with initial values of $V_{B}(0)=\$ 70$ million and $V_{C}(0)=\$ 100$ million; volatilities of them are $\sigma_{B}=\sigma_{1}=0.2, \sigma_{C}=\sigma_{2}=0.3$ respectively; recover rate $R=0.3$; correlation $\rho=0.7 ; \gamma_{i}=0$ and the default barriers are $\$ 40$ million and $\$ 60$ million respectively. 
In Figure 1, method 1 means the CDS spread is obtained by simulating directly on the original Formula (2), method 2 means the CDS spread is calculated by our closed form solution with the integral evaluated by Monte Carlo method. The method 1 is repeated 10000 times using computer time 157.6858 second, while the method 2 is repeated 1000 times using computer time 178.7021 second. We can see that the calculation by our solution converges much faster than directly simulate the original formula. As less as $1 / 10$ times, the result of the method 2 is much better than the method 1 .

Now use the closed form solution, by Monte Carlo simulate 1000 times to calculate the integral, we can analysis the parameters of $R, \rho, \sigma$ and $T$ respectively. The other parameters are chosen as above.

The left figure and the right one show the impact of correlation coefficient $\rho$, maturity time $T$ and recovery rate $R$ on CDS spread. Two figures in Figure 2 show their relationship.

In the upper figure of Figure 2, CDS spreads are greater for swaps with longer maturities. The lower one illustrates the extent to which CDS spreads depend on the recovery rate. When the recovery rate becomes larger, the payoff will get smaller. Hence the CDS spread is getting smaller when recovery rate getting larger. Both of them show that the spread goes down as the correlation goes up.

Figure 3 confirms that CDS spread increases with expired time $T$ and decreases with recover rate $R$, when the correlation is fixed.

Figure 4 show what kind of the rules for the volatilities of the two companies. The behaviors of them affect to the CDS spread in different way. Suppose that the other parameters are fixed. If the volatility of the Company $B$ is larger, which means the probability of the default goes larger as well, it results that the CDS spread is more expansive. On the other hand, if the volatility of the Company $\mathrm{C}$ is larger, which means the probability of the failure of the CDS payoff is larger, it results CDS spread is cheaper.

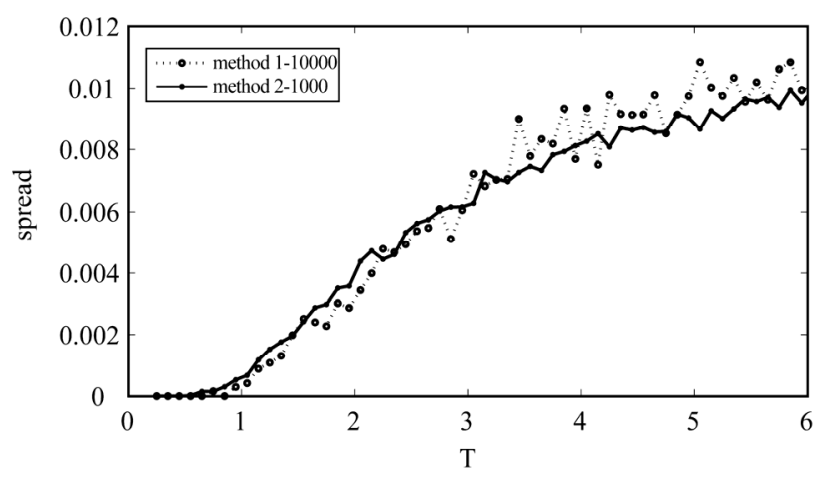

Figure 1. CDS spread with counterparty risk by two methods.
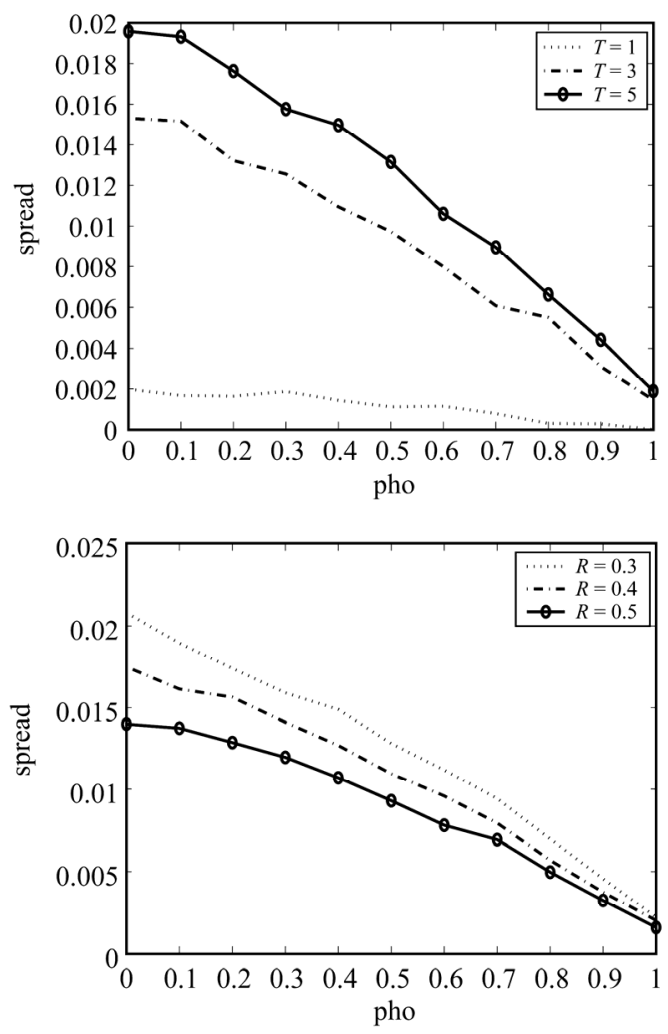

Figure 2. CDS spread with counterparty risk vs. correlation $\rho$, varying $T$ (upper) and $R$ (lower).

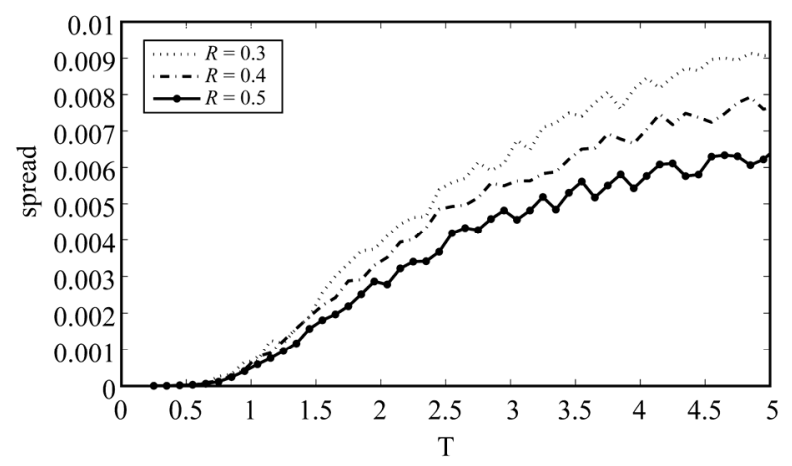

Figure 3. CDS spread with counterparty risk vs. time $T$, varying $R$.

Figure 5 is a three-dimensional surface of the value for the probability of $\left.u\left(V_{B}(0), V_{C}(0), 0\right)\right|_{T}=5$ respect to $V_{B}(0)$ and $V_{C}(0)$.

\section{Conclusions}

In this paper, we have introduced a PDE methodology for modeling default correlations. We assume that the value of companies follow correlated geometric brownian motions. When the asset value of a company reaches a predefined barrier, a credit event called default occurs. 

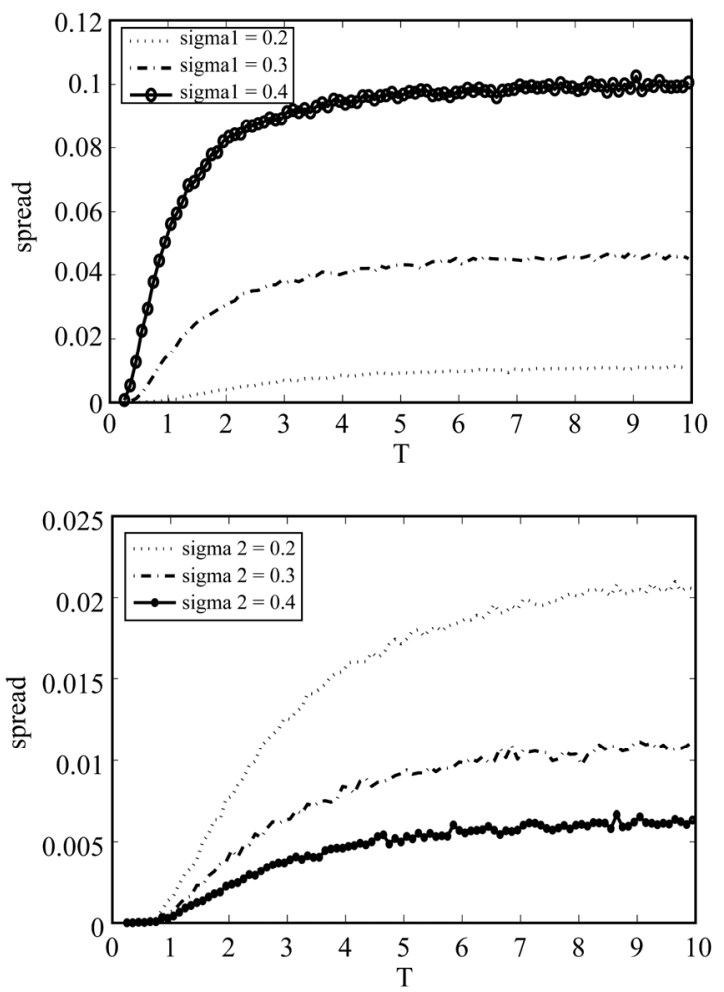

Figure 4. CDS spread with counterparty risk vs. time $T$, varying $\sigma_{1}$ (upper) and $\sigma_{2}$ (lower).

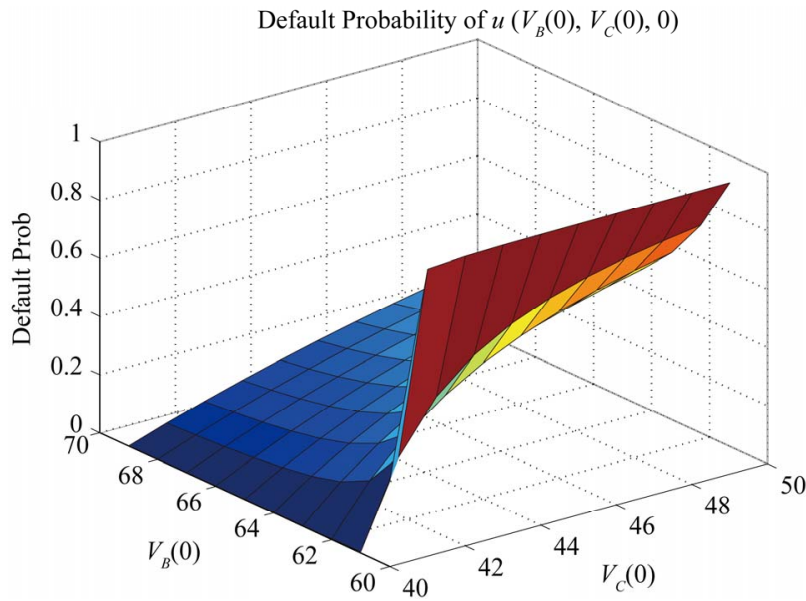

Figure 5. is a three-dimensional surface of the value for the probability of $\left.u\left(V_{B}(0), V_{C}(0), 0\right)\right|_{T}=5$ respect to $V_{B}(0)$ and $V_{B}(\mathbf{0})$.

The essential part is to derive the joint default probability as the solution to a partial differential equation. This solution is more computationally efficient than traditional simulation for original formula or lattice techniques to the equation. We applied the default probabilities solved from the PDE to the valuation of credit default swaps with counterparty default risk. The model can be extended to the valuation of any credit derivative when the payoff is based on defaults by two companies.

The shortage of the model is limited by the dimension, it is difficult to extend the method to a basket CDS with a large portfolio.

\section{Acknowledgements}

The authors would like to express the thanks to Prof. Lishang Jiang for the helpful discussions and suggestions.

\section{References}

[1] R. Merton, "On the Valuing of Corporate Debt: The Risk Structure of Interest Rates," Journal of Finance, Vol. 29, No. 2, 1974, pp. 449-470. doi:10.2307/2978814

[2] F. Black and J. Cox, "Valuing Corporate Securities: Some Effects of Bond Indenture Provisions," Journal of Finance, Vol. 31, No. 2, 1976, pp. 351-367. doi:10.2307/ 2326607

[3] F. Longstaff and E. Schwartz, "A Simple Approach to Valuing Risky Fixed and Floating Rate Debt," Journal of Finance, Vol. 50, No. 3, 1995, pp. 789-819. doi:10.2307/ 2329288

[4] C. Zhou, “A Jump-Diffusion Approach to Modeling Credit Risk and Valuing Defaultable Securities," Finance and Economics Discussion Series, Working Paper, Board of Governors of the Federal Reserve System, Washington DC, 1997.

[5] C. Zhou, "An Analysis of Default Correlation and Multiple Defaults," Review of Finance Studies, Vol. 14, No. 2, 2001, pp. 555-576. doi:10.1093/rfs/14.2.555

[6] C. Zhou, "The Term Structure of Credit Spreads with Jump Risk,” Journal of Banking \& Finance, Vol. 25, No. 11, 2001, pp. 2015-2040. doi:10.1016/S0378-4266(00)00 $168-0$

[7] D. Duffie and K. J. Singleton, "Modeling Term Structures of Defaultable Bonds," Review of Financial Studies, Vol. 12, No. 4, 1999, pp. 687-720. doi:10.1093/rfs/12.4. 687

[8] D. Duffie and K. J. Singleton, “Credit Risk,” Princeton University Press, Princeton, 2003.

[9] D. Lando, "On Cox Processes and Credit Risky Securities,” Review of Derivatives Research, Vol. 2, No. 2-3, 1998, pp. 99-120. doi:10.1007/BF01531332

[10] J. Hull and A. White, "Valuing Credit Default Swaps I: No Counterparty Default Risk," Journal of Derivatives, Vol. 8, No. 1, 2000, pp. 29-40. doi:10.3905/jod.2000. 319115

[11] J. Hull and A. White, "Valuing Credit Default Swaps II: Modeling Default Correlations," Journal of Derivatives, Vol. 8, No. 3, 2001, pp. 12-22. doi:10.3905/jod.2001. 319153

[12] R. Jarrow and Y. Yildirim, “A Simple Model for Valuing Default Swaps when Both Market and Credit Risk are 
Correlated,” Journal of Fixed Income, Vol. 11, No. 4, 2002, pp. 7-19. doi:10.3905/jfi.2002.319308

[13] R. Jarrow and F. Yu, "Counterparty Risk and the Pricing of Defaultable Securities," Journal of Finance, Vol. 56, No. 5, 2001, pp. 1765-1799. doi:10.1111/0022-1082.003 89

[14] F. Yu, "Correlated Defaults and the Valuation of Defaultable Securities," Proceedings of 2nd International Conference on Credit Risk, Montréal, 15-16 April 2004, pp. 1-30.

[15] S. Y. Leung and Y. K. Kwok, "Credit Default Swap Valuation with Counterparty Risk," The Kyoto Economic Review, Vol. 74, No. 1, 2005, pp. 25-45.

[16] R. Jarrow and S. M. Turnbull, "Pricing Derivatives on Financial Securities Subject to Credit Risk," Journal of Finance, Vol. 50, No. 1, 1995, pp. 53-86. doi:10.2307/ 2329239

[17] J. Hull and A. White, "Valuation of a CDO and nth to Default CDS without Monte Carlo Simulation,” Journal of Derivatives, Vol. 12, No. 2, 2004, pp. 8-23. doi:10. 3905/jod.2004.450964

[18] J. Hull, M. Predescu and A. White, "The Relationship between Credit Default Swap Spreads, Bond Yields, and Credit Rating Announcements," Journal of Banking \& Finance, Vol. 28, No. 11, 2004, pp. 2789-2811. doi:10. 1016/j.jbankfin.2004.06.010

[19] J. Hull, M. Predescu and A. White, "The Valuation of
Correlation-Dependent Credit Derivatives Using a Structural Model,” Journal of Credit Risk, Vol. 6, No. 3, 2010, pp. 99-132.

[20] M. Kijima and Y. Muromachi, "Credit Events and the Valuation of Credit Derivatives of Basket Type," Review of Derivatives Research, Vol. 4, No. 1, 2000, pp. 55-79. doi:10.1023/A:1009676412322

[21] M. Kijima and Y. Muromachi, "Valuation of a Credit Swap of the Basket Type," Review of Derivatives Research, Vol. 4, No. 1, 2000, pp. 81-97. doi:10.1023/A: 1009628513231

[22] M. Wise and V. Bhansali, "Correlated Random Walks and the Joint Survival Probability,” Working Paper, Caltech and PIMCO, pp. 1-13.

[23] P. Zhou and J. Liang, "Analysis of Credit Default Swap," Applied Mathematics-JCU, Vol. 22, 2007, pp. 311-314.

[24] K. Giesecke and L. R. Goldberg, "The Market Price of Credit Risk,” Working Paper, Cornell University, New York, 2003, pp. 1-29.

[25] J. Hull, “Options, Futures and Other Derivatives,” 7th Edition, Prentice Education, Upper Saddle River, 2009.

[26] H. He, P. W. Keirstead and J. Rebholz, "Double Lookbacks,” Mathematical Finance, Vol. 8, No. 3, 1998, pp. 201-228. doi:10.1111/1467-9965.00053

[27] I. S. Gradshteyn and I. M. Ryzhik, "Table of Integrals, Series, and Products,” Academic Press, New York, 1980. 